\title{
Long-wavelength approximation for string cosmology with barotropic perfect fluid
}

\author{
Piret Kuusk ${ }^{1}$ \\ Institute of Physics, University of Tartu, Riia 142, Tartu 51014, Estonia \\ Margus Saal ${ }^{2}$ \\ University of Tartu, Tähe 4, Tartu 51010, Estonia \\ PACS number: 98.80 Dr
}

\begin{abstract}
The field equations derived from the low energy string effective action with a matter tensor describing a perfect fluid with a barotropic equation of state are solved iteratively using the long-wavelength approximation, i.e. the field equations are expanded by the number of spatial gradients. In the zero order, a quasi-isotropic solution is presented and compared with the general solution of the pure dilaton gravity. Possible cosmological models are analyzed from the point of view of the pre-big bang scenario. The second order solutions are found and their growing and decaying parts are studied.
\end{abstract}

${ }^{1}$ Electronic address: piret@fi.tartu.ee

${ }^{2}$ Electronic address: margus@hexagon.fi.tartu.ee 


\section{Introduction}

The long-wavelength iteration scheme for investigating the early stages of cosmological models was first introduced by Lifschitz and Khalatnikov [1] in the case of the Einstein equations with pure radiation as a source term. An improved scheme was presented by Tomita [2] where its main assumption was taken to be the smallness of spatial variations of metric $\partial_{l} \gamma_{i j}$ compared with time variations $\partial_{0} \gamma_{i j}$. Then Comer, Deruelle, Langlois, and Parry [3] applied it to the Einstein equations with a perfect fluid matter (with a barotropic equation of state, cf also [4]) and in the case of a scalar field with a potential. The next step was done by Tomita and Deruelle [5] who examined the case of two fluids (an inflationary and a standard fluid). Finally, the scalar-tensor theory with a perfect fluid has been discussed by Comer, Deruelle, and Langlois [6]. The long-wavelength approximation for the Hamilton-Jacobi formalism (the gradient expansion for the generating functional) was developed by Salopek, Stewart, and Parry [7].

In the following, we solve iteratively field equations derived from the low energy string effective action with a perfect fluid matter with additional requirement that the matter obeys a barotropic equation of state. In general, the approximation scheme can be applied in the case of anisotropic and inhomogeneous spacetimes. In the present paper we ignore the local anisotropy, although this assumption may be incorrect near singularity [8]. We find a quasi-isotropic solution for the field equations in the zero approximation (neglecting all spatial gradients) which include both post-big bang and pre-big bang $[9,10,11,12]$ cosmological models. It has been demonstrated $[13,14]$ that a smooth transition from a pre-big bang branch to a post-big bang branch (the graceful exit problem, see also $[15,16,17]$ ) is impossible in this approximation, even if we incorporate some dilaton potential, or cosmological constant. We analyze the nature of discontinuities which are contained in cosmological models based on a quasi-isotropic solution with a barotropic 
perfect fluid as a matter source (cf [18]).

Recently, Khoury, Ovrut, Seiberg, Steinhardt, and Turok [19] presented a new cosmological scenario where the Universe is initially contracting towards a big crunch and then makes a transition through a singularity to the post-big bang Universe. The model is a part of the M-theory motivated ekpyrotic scenario developed by Khoury, Ovrut, Steinhardt, and Turok [20] and occurs in a recent proposal which introduces the cyclic nature of the ekpyrotic Universe [21]. The same considerations can be applied to the reversal problem in the pre-big bang scenario.

The paper is organized as follows. In the next section, the string frame field equations in a synchronous coordinate system are given. In the third section, solutions of the field equations in the zero approximation (taking spatial gradient terms to vanish) in the case of pure dilaton gravity and in the case of dilaton gravity with a perfect fluid matter obeying a barotropic equation of state are presented. Specific interpretations of solutions are considered and problems of cosmological model building are studied. In the fourth section, the second order solutions in the long-wavelength approximation are found. It is analyzed how the equation of state for matter influences the evolution of inhomogeneities. The fifth section is a summary.

\section{$2 \quad$ Field Equations}

Upon compactification the low energy action can be written in the lowest order in the inverse string tension and coupling and using the string (Jordan) frame as

$$
\begin{aligned}
I_{e f f} & =\frac{1}{2 \lambda_{s}^{2}} \int d^{4} x \sqrt{-g} e^{-\phi}\left[{ }^{4} \mathcal{R}+g^{\mu \nu} \partial_{\mu} \phi \partial_{\nu} \phi-\frac{1}{12} H_{\mu \nu \rho} H^{\mu \nu \rho}+V(\phi)\right] \\
& -\int d^{4} x \sqrt{-g}\left[L_{m}-\Lambda\right] .
\end{aligned}
$$


Here ${ }^{4} \mathcal{R}$ is the curvature scalar of the metric $g_{\mu \nu}, \phi$ is the dilaton field determining the strength of the gravitational coupling through $g_{s}^{2} \sim e^{\phi}$, $H_{\mu \nu \rho}$ is an antisymmetric field strength, $\lambda_{s}$ is the fundamental string length scale, $L_{m}$ is the matter lagrangian, and $\Lambda$ is the cosmological constant. We assume that there is no direct coupling between the dilaton and matter fields.

The energy-momentum tensor for a perfect fluid matter is

$$
T_{\mu \nu}=(\rho+p) u_{\mu} u_{\nu}+p g_{\mu \nu}
$$

and we assume that the energy density and pressure are related by a barotropic equation of state

$$
p=(\Gamma-1) \rho, 0 \leq \Gamma \leq 2 .
$$

Here $\Gamma=\frac{4}{3}$ corresponds to a pure radiation, $\Gamma=1$ to a pressureless dust, $\Gamma=\frac{2}{3}$ to an inflationary fluid, $\Gamma=0$ to a phenomenological matter sector cosmological constant, and $\Gamma=2$ to a stiff fluid.

Let us introduce a synchronous gauge

$$
d s^{2}=-d t^{2}+\gamma_{i j}(t, x) d x^{i} d x^{j}
$$

and additional simplifying assumptions of vanishing three-form field, $H_{\mu \nu \rho}=$ 0 , dilaton potential, $V(\phi)=0$, and cosmological constant, $\Lambda=0$. Now the field equations following from the action functional (1) with a barotropic perfect fluid (2), (3) as the matter source can be written

$$
\begin{aligned}
\mathcal{R}_{0}^{0} \equiv \frac{1}{2} \dot{K}+\frac{1}{4} K_{j}^{i} K_{j}^{i} & =\frac{1}{4} e^{\phi} \rho\left(2-3 \Gamma-2 \Gamma u_{s} u^{s}\right)+\frac{1}{4} K \dot{\phi} \\
& +\frac{1}{2}\left(3 \ddot{\phi}-\dot{\phi}^{2}+\partial_{r} \phi \partial^{r} \phi-\nabla_{r} \nabla^{r} \phi\right) \\
\mathcal{R}_{i}^{0} \equiv \frac{1}{2}\left(\nabla_{i} K-\nabla_{j} K_{i}^{j}\right) & =\frac{1}{2} e^{\phi} \rho \Gamma u_{i} \sqrt{1+u_{s} u^{s}}-\frac{1}{2} K_{i}^{j} \nabla_{j} \phi+\nabla_{i} \dot{\phi},(6) \\
\mathcal{R}_{i}^{j} \equiv R_{i}^{j}+\frac{1}{2} \dot{K}_{i}^{j}+\frac{1}{4} K K_{i}^{j} & =\frac{1}{4} e^{\phi} \rho\left[(2-\Gamma) \delta_{i}^{j}+2 \Gamma u_{i} u^{j}\right]+\frac{1}{2} K_{i}^{j} \dot{\phi}
\end{aligned}
$$




$$
\begin{aligned}
& +\frac{1}{4} K \dot{\phi} \delta_{i}^{j}+\frac{1}{2} \delta_{i}^{j}\left(\ddot{\phi}-\dot{\phi}^{2}+\partial_{r} \phi \partial^{r} \phi-\nabla_{r} \nabla^{r} \phi\right)-\nabla_{i} \nabla^{j} \phi, \\
& \ddot{\phi}+\frac{1}{2} K \dot{\phi}-\dot{\phi}^{2}-\nabla_{r} \nabla^{r} \phi+\partial_{r} \phi \partial^{r} \phi=\frac{1}{2} e^{\phi}[(3 \Gamma-4) \rho] .
\end{aligned}
$$

Here $K_{i j}=\dot{\gamma}_{i j}, K=K_{i}^{i}=\gamma^{i j} \dot{\gamma}_{i j}$ and $R_{i}^{j}$ is the Ricci tensor of the threemetric $\gamma_{i j}$. In addition, we have the usual conservation law for the matter density $\nabla_{\mu} T_{\nu}^{\mu}=0$.

\section{Zero Order Solutions}

In the zero order approximation we ignore spatial gradients and local anisotropy in field equations (5)-(8). A solution for the metric is taken to be in a quasi-isotropic form

$$
\gamma_{i j}\left(t, x^{k}\right)=a^{2}(t) h_{i j}\left(x^{k}\right)
$$

where $h_{i j}\left(x^{k}\right)$ is a time independent seed metric and $a(t)$ is a scale factor of an isotropic and homogeneous Universe. As demonstrated in $[2,3]$, the quasi-isotropic solution (9) can be regarded as an attractor of general anisotropic solutions. We will consider only a particular class of solutions with a power law evolution of the scale factor and a logarithmically evolving dilaton

$$
a=a_{0} \tau^{\alpha(\Gamma)}, \quad \phi=\phi_{0}-\beta(\Gamma) \ln \tau,
$$

where $\tau$ is a time parameter. Solutions of this form can be found only in the case of the pure dilaton gravity with a vanishing potential, $V(\phi)=0$, and with a perfect barotropic fluid matter (2), (3) as a source. Inclusion of the cosmological constant $\Lambda$ introduces a hyperbolic nature of solutions and we shall not consider this case here. Solutions (10) inevitably contain singular points $\left(a \rightarrow 0, g_{s}^{2} \sim e^{\phi} \rightarrow \infty\right)$ and it is not possible to avoid them. 


\subsection{Pure Dilaton Gravity}

The field equations for the Hubble parameter $H(t) \equiv \frac{\dot{a}(t)}{a(t)}$ and the dilaton $\phi(t)$ can be written as follows

$$
\begin{aligned}
\dot{H}+3 H^{2}-H \dot{\phi} & =0, \\
6 H^{2}-6 H \dot{\phi}+\dot{\phi}^{2} & =0, \\
\ddot{\phi}+3 H \dot{\phi}-\phi^{2} & =0 .
\end{aligned}
$$

The system is not overdetermined, since only two equations are independent.

The most general isotropic solution reads

$$
\begin{gathered}
a=a_{0}\left(A t+t_{0}\right)^{\frac{B}{\sqrt{3}}}, \\
\phi=\phi_{0}+(B \sqrt{3}-1) \ln \left(A t+t_{0}\right) .
\end{gathered}
$$

Here $B= \pm 1$ denote two branches of solutions. The range of variation of the time coordinate $t$ must be chosen such that $A t+t_{0} \geq 0$, so the time inversion amounts to a change of constant $A \rightarrow-A$. The dilaton gravity analogue of a post-big bang model corresponds to $A>0, B=1$, a standard pre-big bang model $[9,10,11,12]$ corresponds to $A<0, B=-1$. Integration constants $a_{0}, \phi_{0}$ are equal to values of $a(t), \phi(t)$ at an initial moment $t_{i}=\frac{1-t_{0}}{A}$ and $A$ determines initial values for $\dot{a}(t), \dot{\phi}(t)$.

An one-to-one correspondence between post-big bang solutions and prebig bang solutions can be arranged by duality transformations

$$
a(t) \rightarrow \bar{a}(t)=a^{-1}(-t), \quad \phi(t) \rightarrow \bar{\phi}(t)=\phi(-t)-6 \ln a(-t) .
$$

In terms of general solution (14), (15), the correspondence between a particular post-big bang solution $(B=1)$ with $a_{0}, \phi_{0}$ and a pre-big bang solution $(B=-1)$ is encoded in integration constants $\bar{a}_{0}, \bar{\phi}_{0}, A$ that have undergone the same duality transformation (16):

$$
\begin{gathered}
\bar{a}(t)=a_{0}^{-1}\left(t_{0}-A t\right)^{-\frac{1}{\sqrt{3}}} \\
\bar{\phi}(t)=\phi_{0}-6 \ln a_{0}-(\sqrt{3}+1) \ln \left(t_{0}-A t\right) .
\end{gathered}
$$




\subsection{Dilaton Gravity and Perfect Fluid Matter}

After some manipulations the field equations become

$$
\begin{gathered}
\dot{H}+3 H^{2}-H \dot{\phi}=\frac{1}{2} e^{\phi} \rho(\Gamma-1), \\
6 H^{2}-6 H \dot{\phi}+\dot{\phi}^{2}=e^{\phi} \rho, \\
\ddot{\phi}+3 H \dot{\phi}-\dot{\phi}^{2}=\frac{1}{2} e^{\phi} \rho(3 \Gamma-4), \\
\dot{\rho}+3 H \Gamma \rho=0,
\end{gathered}
$$

where the last equation is the conservation law of matter. Loosely speaking, equations (19), (21) and (22) describe the evolution of $H, \phi$ and $\rho$, remaining equation (20) imposes a constraint.

A quasi-isotropic solution of the field equations can be written as follows

$$
\begin{aligned}
a & =a_{0}\left(t_{0} \pm|\chi| t\right)^{\frac{2(\Gamma-1)}{\Delta}} \\
\phi & =\phi_{0}-\frac{2(4-3 \Gamma)}{\Delta} \ln \left(t_{0} \pm|\chi| t\right) \\
\rho & =\rho_{0}\left(t_{0} \pm|\chi| t\right)^{-\frac{6 \Gamma(\Gamma-1)}{\Delta}}
\end{aligned}
$$

where

$$
\begin{gathered}
\chi \equiv \pm \sqrt{\frac{e^{\phi_{0}} \rho_{0}(\Delta)^{2}}{-4(\Delta-2)}}, \\
\Delta \equiv 3(\Gamma-1)^{2}+1>0,
\end{gathered}
$$

and $a_{0}, \phi_{0}, \rho_{0}$ are integration constants which are equal to the values of functions $a(t), \phi(t), \rho(t)$ at an initial moment $t_{i}=\frac{1-t_{0}}{ \pm|\chi|}$. The solution is not the most general one, since initial values of $\dot{a}(t)$ and $\dot{\phi}(t)$ are both uniquely determined by barotropic index $\Gamma$ and the initial values of $\phi(t)$ and $\rho(t)$; in the most general case they must contain an additional arbitrary integration constant. As a result, solution (23)-(27) turns out to be singular at $\Gamma_{s}=1 \pm \frac{1}{\sqrt{3}}$, where $\Delta-2=0, \chi \rightarrow \infty$. Note that in the case of 
unusal matter with $(\Gamma-1)^{2}>1 / 3$ we must take $\rho_{0}<0$. For the zero order three-velocity $u_{i}$, field equation (6) implies $u_{i} \equiv 0$. The range of variation of the time coordinate $t$ must be chosen such that $t_{0} \pm|\chi| t \geq 0$; $t_{0}$ is a numerical constant corresponding to the freedom of constant shift in time. In comparison with solutions of the pure dilaton gravity (14)-(15), the integration constant $A$ is replaced by constant $\chi=\chi\left(\rho_{0}, \phi_{0}, \Gamma\right)$ and the choice of the branch, $B= \pm 1$, is imitated by the choice of barotropic index $\Gamma$, or more exactly, by the sign of expression $\Gamma-1 \in[-1,+1]$.

Solution (23) for $a(t)$ determines the Hubble parameter as

$$
H=\frac{ \pm|\chi|}{\left(t_{0} \pm|\chi| t\right)} \frac{2}{\Delta}(\Gamma-1), \quad \dot{H}=\frac{-\chi^{2}}{\left(t_{0} \pm|\chi| t\right)^{2}} \frac{2}{\Delta}(\Gamma-1) .
$$

We see that constant $\chi$ is proportional to the initial value of the Hubble parameter at the initial moment $t_{i}, t_{0} \pm|\chi| t_{i}=1$, and in the case of matter with barotropic index $\Gamma_{s}=1 \pm \frac{1}{\sqrt{3}}$ it diverges. In what follows we don't consider barotropic indices belonging to the neighborhood of $\Gamma_{s}$.

If we take $\Gamma>1$ and temporal argument $\left(t_{0}+|\chi| t\right)$ we get a decelerating post-big bang Universe, e.g. for $\Gamma=\frac{4}{3}$ (radiation dominated stage) we have $|\chi|=\sqrt{\frac{2}{3} e^{\phi_{0}} \rho_{0}}$ and $a(t)=a_{0}\left(t_{0}+|\chi| t\right)^{\frac{1}{2}}$, matter density is decreasing, $\rho=\rho_{0}\left(t_{0}+|\chi| t\right)^{-2}$, and dilaton is freezed to a constant, $\phi=\phi_{0}$. Integration constants $a_{0}$ and $\rho_{0}$ are (initial) values of corresponding variables at the moment $t_{i}=\frac{1-t_{0}}{|\chi|}$. A singularity $(a \rightarrow 0, \rho \rightarrow \infty)$ is reached at the moment $t_{s}=-\frac{t_{0}}{|\chi|}$. The domain of the time variable is $t \in\left(t_{s},+\infty\right)$. Taking into account our choice of initial moment $t_{i}$ we see that $t_{i}=t_{s}+\frac{1}{|\chi|}$, i.e. $\frac{1}{|\chi|}$ is the time interval from the singularity to the initial moment. If $t_{0}>0$ we have $t_{s} \leq t_{i} \leq 0$ and the Universe is regular at the moment $t=0$. If the source matter is exotic $(\Gamma<1)$, a solution $(23)-(25)$ represents a contracting Universe beginning from a singularity at $t_{s}$ and going through $t_{i}$.

The pre-big bang branch of solutions corresponds to the minus sign in front of $|\chi| t$ and exotic matter $\Gamma-1<0$. The domain of the time coordinate is $t \in\left(-\infty, \frac{t_{0}}{|\chi|} \equiv t_{\infty}\right)$, i.e. $t_{0}-|\chi| t>0$, and the behaviour of the scale factor 
is superinflationary $(\dot{a}>0, \ddot{a}>0, H>0, \dot{H}>0)$. Integration constants $a_{0}$, $\phi_{0}$, and $\rho_{0}$ are the values of corresponding variables at the moment $t_{i} \equiv \frac{1-t_{0}}{-|\chi|}$ and here $t_{i}=t_{s}-\frac{1}{|\chi|}$, i.e. $\frac{1}{|\chi|}$ is the time interval from the initial moment to the singularity. A contracting solution with a future singularity is obtained if $\Gamma>1$.

Expressions (24) for $\phi$ and (28) for $H$ imply

$$
H=-(\Gamma-1) \dot{\bar{\phi}}, \quad \dot{\bar{\phi}} \equiv \dot{\phi}-3 H
$$

and the behaviour of solutions depending on barotropic index $\Gamma$ can be summarized on a phase diagram analogous to the corresponding one familiar from the pre-big bang scenario of the pure dilaton gravity:

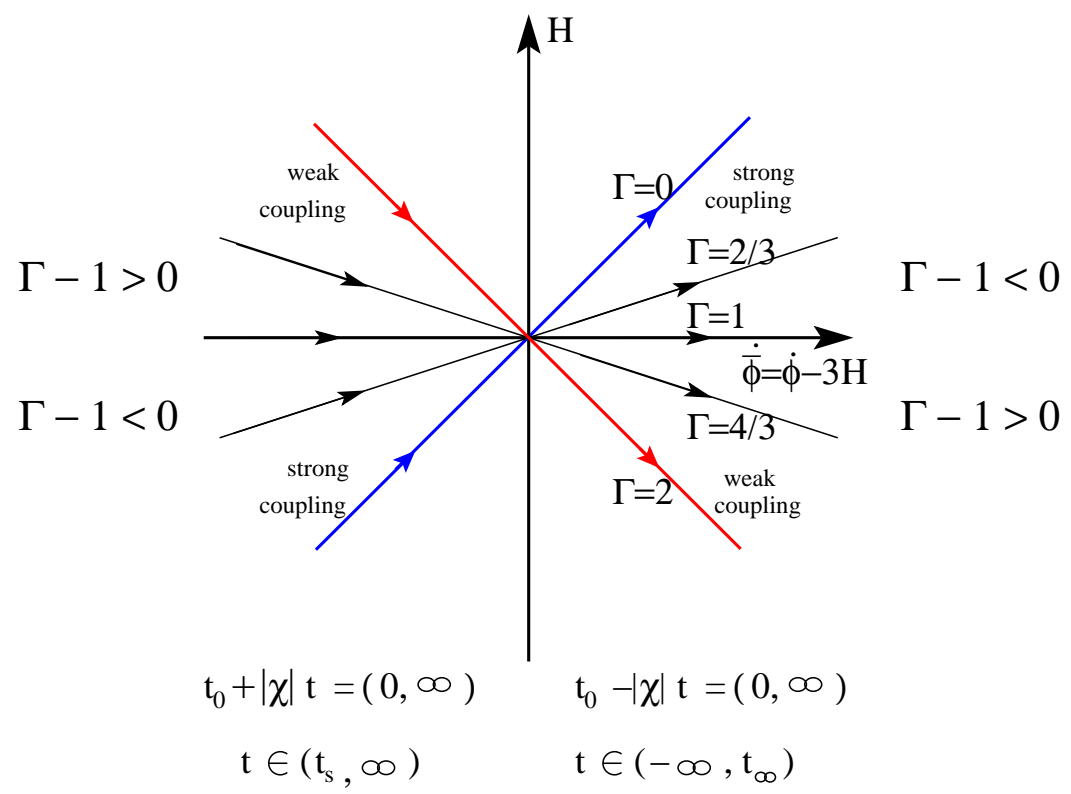

As in the case of pure dilaton gravity, solution (23)-(25) contains both the post-big bang and the pre-big bang branch. The latter ones can be related to the former ones by duality transformations (16), but in order to 
satisfy the field equations, here we need an additional transformation for barotropic index $\Gamma$ and for energy density $\rho$ :

$$
\frac{p}{\rho} \rightarrow \frac{\bar{p}}{\bar{\rho}}=-\frac{p}{\rho} \Longrightarrow \bar{\Gamma} \rightarrow 2-\Gamma, \quad \rho \rightarrow \rho a^{6}(-t) .
$$

The corresponding pre-big bang solutions (i.e. with the time dependence in the form $\left.\left(t_{0}-|\chi| t\right)\right)$ now read

$$
\begin{aligned}
& \bar{a}=a_{0}^{-1}\left(t_{0}-|\chi| t\right)^{\frac{2(\bar{\Gamma}-1)}{\Delta}}, \\
& \bar{\phi}=\phi_{0}-6 \ln a_{0}-\frac{2(4-3 \bar{\Gamma})}{\Delta} \ln \left(t_{0}-|\chi| t\right), \\
& \bar{\rho}=\rho_{0} a_{0}^{6}\left(t_{0}-|\chi| t\right)^{-\frac{6 \bar{\Gamma}(\overline{\bar{\Gamma}}-1)}{\Delta}} .
\end{aligned}
$$

Note that $\rho_{0} e^{\phi_{0}}=\bar{\rho}_{0} e^{\bar{\phi}_{0}}, \Delta=\bar{\Delta}$ and $t_{0}$ don't change under duality transformations (16), (30).

\subsection{Model building}

In the case of pure dilaton gravity, the main idea of the original pre-big bang cosmology was to use a superinflationary pre-big bang branch obtained from a decelerating post-big bang branch by duality transformations for constructing a cosmological model valid in the time range $t \in(-\infty,+\infty)$. However, due to rather general no-go theorems [14] a smooth connection of these two branches turned out to be impossible, at least without some kind of higher order corrections. In the case of a perfect barotropic fluid, mutually dual solutions (23)-(25) and (31)-(33) contain different barotropic indices $\Gamma, \bar{\Gamma}=2-\Gamma$ and consequently also different matter sources. This means that if we try to repeat the strategy of the original pre-big bang cosmology, we must admit a profound change in the type of cosmological matter and join a pre-big bang solution with barotropic index $(2-\Gamma)$ with a solution with barotropic index $\Gamma$ ( $\mathrm{cf}[17,18])$. We are free to choose the moment of branch change, but the explicit solution (28) for $H$ indicates that we cannot achieve continuity for both $H$ and $\dot{H}$. 
For example, let $a_{0}=1$ and $t_{0}=1$ and let the junction moment be the regular point $t=0$. From the solutions (23)-(25), (31)-(33) it is easy to see that $a(t), \dot{a}(t), \phi(t)$ and $\rho(t)$ are continuous there, but not $\dot{H}(t)$ (or $\ddot{a}(t)$ ), $\dot{\phi}(t)$ and $\dot{\rho}(t)$ :

$$
\begin{gathered}
\dot{\bar{H}}(0)=2 \chi^{2}(\Gamma-1) \Delta^{-1}=-\dot{H}(0), \quad \dot{\bar{\phi}}(0)=\dot{\phi}(0)-4|\chi| \Delta^{-1}, \\
\dot{\bar{\rho}}(0)=\dot{\rho}(0)+12 \rho_{0}|\chi|(\Gamma-1)^{2} \Delta^{-1}
\end{gathered}
$$

It is possible, that these kinematical discontinuities could be given in terms of physical changes of matter sources. However, a dynamical description of these changes remains open. Numerical values of discontinuities depend on the numerical value of the constant $\chi$ given in terms of barotropic index $\Gamma$ and initial values of the dilaton and matter density (26). We can choose $\frac{1}{|\chi|} \sim$ $L_{\text {Planck }}$; then discontinuities are nearly vanishing, but initial moments are almost in the singularities and higher order corrections must be taken into account. Alternatively, we can choose initial moments to be in a classically regular region, far from the singularities; then, however, discontinuities are finite.

The other possibility is to abandon duality transformations altogether and to connect solutions with the same barotropic index $\Gamma$, but with different temporal arguments $\left(t_{0} \pm|\chi| t\right)$ and with arbitrary integration constants $a_{0}$, $\phi_{0}, \rho_{0}, \chi$ and $t_{0}$ (cf ekpyrotic models $[19,20]$ ). However, according to the solution (28) if $\dot{H}$ is continuous, $H$ is not.

As already mentioned, the moment of branch change can be chosen arbitrarily and it may be a regular or a singular point of the solution. This means that the problem of graceful exit is distinct from the problem of singularity. The graceful exit problem in the string cosmology is in fact very similar to that of ordinary inflationary cosmology, where accelerating inflationary stage must be joined with a decelerating FRW model. In ekpyrotic models, discontinuities are concealed in the singularity. 


\section{Second Order Solutions}

Let us we adopt the procedure presented in $[3,6]$ to equations (5)-(8) derived from the string effective action (1). The second order solutions for the threemetric, dilaton, energy density and three-velocity are assumed to be in the form

$$
\begin{aligned}
\gamma_{i j}\left(t, x^{k}\right)=a^{2}(t)\left[h_{i j}+f_{2}(t)\right. & \left.R_{i j}\left(h_{k l}\right)+\frac{1}{3}\left(g_{2}(t)-f_{2}(t)\right) R\left(h_{k l}\right) h_{i j}\right], \\
\phi\left(t, x^{k}\right) & =\phi(t)+\phi_{2}(t) R\left(h_{k l}\right), \\
\rho\left(t, x^{k}\right) & =\rho(t)+\rho_{2}(t) R\left(h_{k l}\right) \\
u_{i}\left(t, x^{k}\right) & =u(t) \nabla_{i} R\left(h_{k l}\right) .
\end{aligned}
$$

Here, $R_{i j}\left(h_{k l}\right)$ and $R\left(h_{k l}\right)$ are the 3-dimensional Ricci tensor and the scalar curvature formed from the seed metric $h_{k l}$, respectively. The zero order solutions $a(t), \phi(t)$, and $\rho(t)$ are given in the previous section. Substituting them into field equations, keeping only terms up to the second order in spatial gradients, collecting coefficients at $R_{i}^{j}$, and $R$ we get a system of ordinary differential equations for the second order corrections $f_{2}, g_{2}$, and $\phi_{2}$.

\subsection{Pure Dilaton Gravity}

If we substitute expressions (36)-(37) into field equations (5)-(8) and take $\rho=0$ we get equations for the second order corrections $f_{2}(t), g_{2}(t)$, and $\phi_{2}(t)$

$$
\begin{gathered}
\ddot{f}_{2}+(3 H-\dot{\phi}) \dot{f}_{2}=-2 a^{-2}, \\
(2 H-\dot{\phi}) \dot{g}_{2}-(6 H-2 \dot{\phi}) \dot{\phi}_{2}=-a^{-2}, \\
\ddot{\phi}_{2}+(3 H-2 \dot{\phi}) \dot{\phi}_{2}+\frac{1}{2} \dot{\phi} \dot{g}_{2}=0 .
\end{gathered}
$$


Upon substituting zero order solutions (23)-(25) into equations (40)-(42) we get the second order solutions (we have taken $A=1$ )

$$
\begin{aligned}
& f_{2}^{ \pm}(t)=c_{1}^{ \pm}+c_{2}^{ \pm} \ln \left(t_{0} \pm t\right)-\frac{3(\sqrt{3} \pm 1)}{4(\sqrt{3} \mp 1)}\left(t_{0} \pm t\right)^{2 \mp \frac{2}{\sqrt{3}}} \\
& \phi_{2}^{ \pm}(t)=e_{1}^{ \pm}+\frac{\sqrt{3} \mp 1}{2 \sqrt{3}} d_{2}^{ \pm}\left(t_{0} \pm t\right)^{-1}+\frac{3 \sqrt{3}}{4(11 \mp 5 \sqrt{3})}\left(t_{0} \pm t\right)^{2 \mp \frac{2}{\sqrt{3}}} \\
& g_{2}^{ \pm}(t)=d_{1}^{ \pm}+d_{2}^{ \pm}\left(t_{0} \pm t\right)^{-1}-\frac{3(4 \sqrt{3} \mp 7)}{2(21 \sqrt{3} \mp 37)}\left(t_{0} \pm t\right)^{2 \mp \frac{2}{\sqrt{3}}}
\end{aligned}
$$

The first terms in expressions (43)-(45) are solutions of corresponding homogeneous equations and the last ones are particular solutions of inhomogeneous equations.

As indicated by Comer, Deruelle and Langlois [6] terms proportional to $\left(t_{0} \pm t\right)^{-1}$ which diverge at $\left(t_{0} \pm t\right) \rightarrow 0$ can be removed by an infinitesimal coordinate transformation that preserves the synchronous coordinate system. In the second approximation, the transformation of the three-metric can be given as $\gamma_{i j}^{\prime}=\gamma_{i j}-2 a \dot{a} T_{2} R\left(h_{i j}\right) h_{i j}$ and the transformation of the dilaton as $\phi^{\prime}=\phi-\dot{\phi} T_{2} R$ with $T_{2}=$ const [6]. If we take $T_{2}=\frac{d_{2}^{ \pm}}{2 \sqrt{3}}$, then terms proportional to $d_{2}^{ \pm}\left(t_{0} \pm t\right)^{-1}$ cancel. The logarithmic term in $f_{2}$ cannot be removed by a coordinate transformation (an analogous problem occurs in the case of linear perturbation theory [22]), but probable it can be treated by means of a renormalization procedure [23].

In the case of lower signs (pre-big bang) solution (43)-(45) coincides with the solution of the corresponding Hamilton-Jacobi equation in the second approximation presented by us earlier and used for estimating the size of an initial homogeneous domain for getting enough inflation in the pre-big bang stage $[24]$. 


\subsection{Dilaton gravity and perfect fluid matter}

Following the procedure described above and eliminating the matter density $\rho$ from field equations we get a system of equations for $f_{2}(t), g_{2}(t)$, and $\phi_{2}(t)$

$$
\begin{gathered}
\ddot{f}_{2}+(3 H-\dot{\phi}) \dot{f}_{2}=-2 a^{-2}, \\
\ddot{g}_{2}+\left[6 H-\frac{(7-6 \Gamma)}{(4-3 \Gamma)} \dot{\phi}\right] \dot{g}_{2}-\left[\frac{6(7-6 \Gamma)}{4-3 \Gamma} H-\frac{12(1-\Gamma)}{(4-3 \Gamma)} \dot{\phi}\right] \dot{\phi}_{2} \\
-\frac{6(1-\Gamma)}{4-3 \Gamma} \ddot{\phi}_{2}=-2 a^{-2}, \\
\ddot{g}_{2}+\left[2 H-\frac{1}{(4-3 \Gamma)} \dot{\phi}\right] \dot{g}_{2}-\left[\frac{6}{4-3 \Gamma} H-\frac{4}{(4-3 \Gamma)} \dot{\phi}\right] \dot{\phi}_{2} \\
-\quad \frac{(10-6 \Gamma)}{4-3 \Gamma} \ddot{\phi}_{2}=0 .
\end{gathered}
$$

These equations are apparently singular at $\Gamma=\frac{4}{3}$ but in fact they are not because of the form of the solution in the zero approximation.

Let us substitute the first order solutions (23)-(25) into equations (46)(48). Upon long but straightforward calculations we obtain the following system of equations

$$
\begin{aligned}
\ddot{f}_{2}^{ \pm} & \pm \frac{2}{\Delta}|\chi|\left(t_{0} \pm|\chi| t\right)^{-1} \dot{f}_{2}^{ \pm}=-2 a_{0}^{-2}\left(t_{0} \pm|\chi| t\right)^{-\frac{4(\Gamma-1)}{\Delta}} \\
\ddot{g}_{2}^{ \pm} & \pm 2|\chi|\left(t_{0} \pm|\chi| t\right)^{-1} \dot{g}_{2}^{ \pm}=(3 \Gamma-5) a_{0}^{-2}\left(t_{0} \pm|\chi| t\right)^{-\frac{4(\Gamma-1)}{\Delta}} \\
\ddot{\phi}_{2}^{ \pm} & \pm \frac{2}{\Delta}|\chi|\left(t_{0} \pm|\chi| t\right)^{-1} \dot{\phi}_{2}^{ \pm}-\frac{4-3 \Gamma}{10-6 \Gamma} \ddot{g}_{2}^{ \pm} \\
& \mp \frac{(2 \Gamma-1)(4-3 \Gamma)}{(5-3 \Gamma) \Delta}|\chi|\left(t_{0} \pm|\chi| t\right)^{-1} \dot{g}_{2}^{ \pm}=0 .
\end{aligned}
$$

The upper sign corresponds to the post-big bang behaviour and the lower sign corresponds to the pre-big bang behaviour. Retaining all integration 
constants general solutions of equations (49)-(51) can be written

$$
\begin{aligned}
& f_{2}^{ \pm}(t)=c_{1}^{ \pm}+c_{2}^{ \pm}\left(t_{0} \pm|\chi| t\right)^{\frac{3 \Gamma^{2}-6 \Gamma+2}{\Delta}} \\
&-\frac{\Delta^{2}|\chi|^{-2} a_{0}^{-2}}{\left(3 \Gamma^{2}-8 \Gamma+6\right)\left(3 \Gamma^{2}-10 \Gamma+10\right)}\left(t_{0} \pm|\chi| t\right)^{2-\frac{4(\Gamma-1)}{\Delta}} \\
& g_{2}^{ \pm}(t)=d_{1}^{ \pm}+d_{2}^{ \pm}\left(t_{0} \pm|\chi| t\right)^{-1} \\
&+\frac{(6 \Gamma-10) \Delta^{2}|\chi|^{-2} a_{0}^{-2}}{4\left(3 \Gamma^{2}-8 \Gamma+6\right)\left(9 \Gamma^{2}-22 \Gamma+16\right)}\left(t_{0} \pm|\chi| t\right)^{2-\frac{4(\Gamma-1)}{\Delta}} \\
& \phi_{2}^{ \pm}(t)=e_{1}^{ \pm}+e_{2}^{ \pm}\left(t_{0} \pm|\chi| t\right)^{\frac{3 \Gamma^{2}-6 \Gamma+2}{\Delta}}+\frac{3 \Gamma-4}{6(\Gamma-1)} d_{2}^{ \pm}\left(t_{0} \pm|\chi| t\right)^{-1} \\
&+\frac{(3 \Gamma-4)\left(3 \Gamma^{2}-6 \Gamma+6\right) \Delta^{2}|\chi|^{-2} a_{0}^{-2}}{4\left(3 \Gamma^{2}-8 \Gamma+6\right)\left(9 \Gamma^{2}-22 \Gamma+16\right)\left(3 \Gamma^{2}-10 \Gamma+10\right)}\left(t_{0} \pm|\chi| t\right)^{2-\frac{4(\Gamma-1)}{\Delta}} .
\end{aligned}
$$

For the matter density correction $\rho_{2}$ we get from equation (8)

$$
\rho_{2}=\frac{2}{(3 \Gamma-4)} e^{-\phi}\left[\ddot{\phi}_{2}+3 H \dot{\phi}_{2}+\frac{1}{2} \dot{g}_{2} \dot{\phi}+2 \dot{\phi}_{\dot{\phi}}-\frac{1}{2}(3 \Gamma-4) e^{\phi} \rho \phi_{2}\right]
$$

and for the three-velocity we get from equation (6)

$$
u(t)=\frac{2 e^{-\phi}}{\Gamma \rho}\left[\frac{1}{3} \dot{g}_{2}-\frac{1}{12} \dot{f}_{2}-H \phi_{2}-\dot{\phi}_{2}\right] .
$$

For the density contrast we can write the expression as follows

$$
\begin{aligned}
\delta= & \frac{\rho_{2}}{\rho}=\frac{2}{(3 \Gamma-4) \rho_{0} e^{\phi_{0}}}\left(t_{0} \pm|\chi| t\right)^{2} \times \\
& {\left[\ddot{\phi}_{2}+3 H \dot{\phi}_{2}+\frac{1}{2} \dot{g}_{2} \dot{\phi}+2 \dot{\phi}_{2}-\frac{1}{2}(3 \Gamma-4) e^{\phi} \rho \phi_{2}\right] . }
\end{aligned}
$$

Let us consider the behaviour of inhomogeneities in the pre-big bang case near the final singularity $\left(|\chi| t \rightarrow t_{0}\right)$. Terms proportional to $\left(t_{0}-\right.$ $|\chi| t)^{\frac{3 \Gamma^{2}-6 \Gamma+2}{\Delta}}$ are decaying if $\Gamma<1-\frac{1}{\sqrt{3}}$ and $\Gamma>1+\frac{1}{\sqrt{3}}$, e.g. at $\Gamma=0$ and $\Gamma=2$. Terms proportional $\left(t_{0}-|\chi| t\right)^{2-\frac{4(\Gamma-1)}{\Delta}}$ are decaying at all values of $\Gamma$. Terms proportional to $\left(t_{0}-|\chi| t\right)^{-1}$ can be treated as in the case 
of pure dilaton gravity: they can be cancelled by an analogous coordinate transformation with $T_{2}= \pm \frac{\Delta d_{2}^{ \pm}}{12(\Gamma-1)|\chi|}$.

In the post-big bang case, the model with $\Gamma=\frac{4}{3}$ should describe a radiation dominated FRW cosmology and so it does in the zero approximation. However, in the second approximation the initial inhomogeneities are not decaying at late times $t \gg t_{0}$, although logarithmically divergent terms that occur in the solution for the pure dilaton gravity are absent here. The full expression for the metric (36) includes terms proportional to $\left(t_{0}+|\chi| t\right)^{2}$ which are growing at all values of $\Gamma$. An analogous term also appears in solutions of field equations corresponding to other models [3], [6], [4]. Khalatnikov, Kamenshchik, and Starobinsky [4] have recently indicated its inevitability near the singularity. However, in our solution and in the solutions presented in [3] it also appears in the late time evolution.

\section{Summary}

In this paper we investigated cosmological solutions of equations (5)-(8), derived from the low energy effective string action, in the framework of long-wavelength approximation. The zero order solution (23)-(27) contains both the pre-big bang and the post-big bang branch, but as it is well known, it is not possible to connect these two branches smoothly. We have found, that the problem of singularity and the problem of graceful exit may be disconnected. The graceful exit problem resembles the problem already known from the first models of inflation: how to get a smooth transition from an accelerating background to a decelerating background. The transition moment and the moment when classical solutions become singular may be different, in general. On the other hand, as indicated in the context of ekpyrotic scenario the occurrence of singularity is strictly a four-dimensional phenomenon and may be used to hide a nonsmooth transition.

The second order corrections to the long-wavelength solutions include 
the effect of spatial gradients. As expected, the picture is symmetric: terms which are growing in the pre-big bang phase are decaying in the post-big bang phase and vice versa; we might use different terms in different asymptotic regimes to estimate the significance of corrective terms. We conclude that in the case of an exotic matter with barotropic index $\Gamma=0$ or $\Gamma=2$ we can achieve the decay of all second order correction terms at the end of the pre-big bang stage. However, corrections for the post-big bang stage always include terms which are growing in time. Our conclusion indicates that a simple model without any dilaton potential or cosmological constant leads to a result which can hardly fit our present understandings. Inclusion of additional terms in the field equations (e.g. $V(\phi), \Lambda$, or $H_{\mu \nu \rho}$ ) changes the form of solutions significally and in that case the analysis of the second order is far more complicated.

\section{ACKNOWLEDGEMENTS}

This work was supported by the Estonian Science Foundation under grant No 5026.

\section{References}

[1] Lifschitz, E. M., and Khalatnikov, I. M., (1963). Adv. Phys. 12, 185.

[2] Tomita, K., (1993). Phys. Rev. D48, 5634.

[3] Comer, G. L., Deruelle, N., Langlois, D. and Parry, J. (1994). Phys. Rev. D49, 2759.

[4] Khalatnikov, I. M., Kamenshchik, A. Yu., and Starobinsky, A. A. (2002) E-print gr-qc/0204045.

[5] Tomita, K., and Deruelle, N. (1994). Phys. Rev. D50, 7216. 
[6] Comer, G. L., Deruelle, N., and Langlois, D. (1997). Phys. Rev. D55 3497.

[7] Salopek, D. S., and Stewart, J. M. (1992) Class. Quantum Grav. 9, 1943; Perry, J., Salopek, D. S., and Stewart, J. M. (1994) Phys. Rev. D49, 2872.

[8] Deruelle, N., and Langlois, D. (1995). Phys. Rev. D52, 2007.

[9] A collection of papers on the pre-big bang scenario is available at homepage http://www.to.infn.it/ gasperin/.

[10] Lidsey, J. E., Wands, D., and Copeland, E. J. (2000). Phys. Rept. 337, 343.

[11] Veneziano, G. (1991) Phys. Lett. B265, 287.

[12] Gasperini, M. and Veneziano, G. (1993) Astropart. Phys. 1, 317.

[13] Brustein, R. and Veneziano, G. (1994). Phys. Lett. B329, 429.

[14] Kaloper, M., Madden, R., and Olive, K. A. (1995) Nucl. Phys. B452, 677.

[15] Gasperini, M., Maggiore, M., and Veneziano, G. (1997). Nucl. Phys. B494, 315; Brustein, R., and Madden, R. (1998). Phys. Rev. D57, 712.

[16] Gasperini, M., Maharana, J., and Veneziano, G. (1996). Nucl. Phys. B472, 349; Dabrowski, M. P., and Kiefer, C. (1997). Phys. Lett. B397, 185.

[17] Ellis, G. F. R, Roberts, D. C., Solomons, D., and Dunsby, P. K. S. (2000) Phys. Rev. D62 084004.

[18] Infante, M. P. and Sánchez, N. (2000) Current Topics in Astrofundamental Physics: the Cosmic Microwave Background (VII Chalogne School, NATO ASI Series C, Kluwer). 
[19] Khoury, J., Ovrut, B. A., Seiberg, N., Steinhardt, P. J., and Turok, N. (2002) Phys. Rev. D65, 086007.

[20] Khoury, J., Ovrut, B. A., Steinhardt, P. J., and Turok, N. (2001) Phys. Rev. D64, 123522.

[21] Steinhardt, P. J. and Turok, N. (2002) Cosmic Evolution in a Cyclic Universe, http://feynman.princeton.edu/ steinh .

[22] Brustein, R., Gasperini,M., Giovannini, M., Mukhanov,V. F., and Veneziano, G. (1995) Phys. Rev. D51, 6744.

[23] Nambu, Y. and Yamaguchi, Y. Y. (1999) Phys. Rev D60, 104011; Nambu, Y. (2000) Phys. Rev D62, 104010; Nambu, Y. (2001) Phys. Rev D63, 044013.

[24] Kuusk, P. and Saal, M. (2002) Gen. Rel. Grav. 34, 353. 